\title{
Search for new physics via photon polarization of
} $b \rightarrow s \gamma$

\section{Naoyuki Haba, ${ }^{a}$ Hiroyuki Ishida, ${ }^{a}$ Tsuyoshi Nakaya, ${ }^{b}$ Yasuhiro Shimizu ${ }^{c, d}$ and Ryo Takahashi ${ }^{a}$}

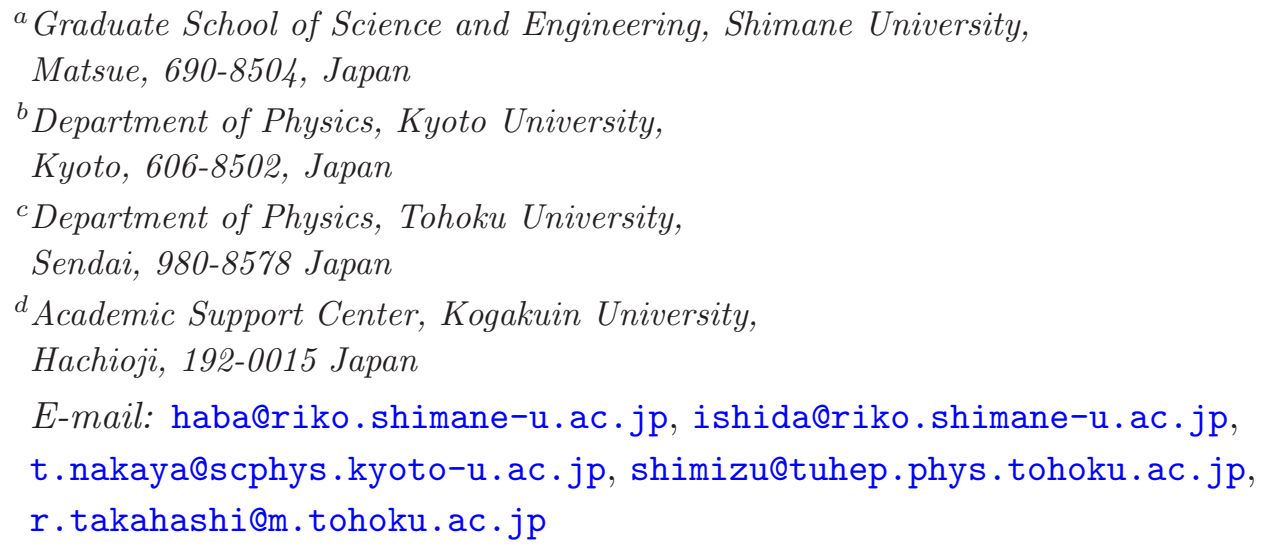

ABSTRACT: We suggest a discriminant analysis of new physics beyond the standard model through a detection of photon polarization in a radiative B meson decay. This analysis is investigated in SUSY SU(5) GUT with right-handed neutrino and left-right symmetric models. New physics search via CP asymmetry in the same process are also evaluated in each model for comparison. We show that new physics can be found via detecting the photon polarization in a parameter space of $\mathrm{TeV}$ energy scale.

Keywords: Rare Decays, Beyond Standard Model, B-Physics

ARXIV EPRINT: 1501.00668 


\section{Contents}

1 Introduction 1

2 Photon polarization 3

2.1 Case of SUSY SU(5) with the right-handed neutrinos 4

2.1.1 Model 4

2.1.2 Photon polarization in SUSY SU(5) with right-handed neutrino 5

2.2 Case of Left-right symmetric standard model 8

2.2.1 Model 8

2.2.2 Photon polarization in LRSM 9

$\begin{array}{lll}3 & \text { CP asymmetry } & 10\end{array}$

$\begin{array}{lll}3.1 & \text { Direct CP asymmetry } & 10\end{array}$

3.1.1 SUSY SU(5) with right-handed neutrino case 11

$\begin{array}{lll}3.1 .2 & \text { LRSM case } & 12\end{array}$

$\begin{array}{ll}3.2 & \text { Time-dependent CP asymmetry } \\ & 13\end{array}$

$\begin{array}{lll}\text { 3.2.1 SUSY SU(5) with right-handed neutrino case } & 13\end{array}$

$\begin{array}{lll}3.2 .2 & \text { LRSM case } & 13\end{array}$

4 Comparison between Photon polarization and Measurements of CP $\begin{array}{ll}\text { asymmetry } & 14\end{array}$

$\begin{array}{lll}5 & \text { Summary } & 15\end{array}$

$6 \quad$ Loop functions $\quad 15$

$\begin{array}{lll}7 & \text { Mass insertion parameters } & 16\end{array}$

\section{Introduction}

In this decade, we have never seen an exotic elementary particle except for the most likely to the standard model (SM) Higgs boson which was discovered at the LHC experiment. This fact might imply that a scale of new physics (NP) is higher than the energy scale that the LHC can reach. Thus, it is important to consider the possibility that such exotics are hard to be directly produced by collider experiments even though the next LHC will become $14 \mathrm{TeV}$.

On the other hand, indirect searches become powerful way to explore the existence of NP and related phenomena beyond the SM even if a new particle is impossible to be produced directly. One popular approach is flavor physics. For instance, flavor changing processes such as $\mu \rightarrow e \gamma$ and $b \rightarrow s \gamma$ retain much information about NP. Models of supersymmetric grand unified theory (SUSY GUT) predict relatively large branching ratios 
of $\operatorname{Br}(\mu \rightarrow e \gamma) \sim \mathcal{O}\left(10^{-15}-10^{-13}\right)$ for the NP scale (the right-handed selectron mass) of $M_{\mathrm{NP}} \simeq(100-300) \mathrm{GeV}$ in an $\mathrm{SU}(5)$ case and $\mathrm{Br}(\mu \rightarrow e \gamma) \sim \mathcal{O}\left(10^{-13}-10^{-11}\right)$ in an $\mathrm{SO}(10)$ case $[1,2]$. Then, ref. [3] suggested that the measurement of the angular distribution of $e$ with respect to the spin direction of the muon in the $\mu \rightarrow e \gamma$ process might distinguish among several extensions of the SM if the signal could be detected. This implies that the precise determination of the chirality of the final $e$ state in the $\mu \rightarrow e \gamma$ process might become a clue to obtain the evidence of NP. This situation is adopted to the $b \rightarrow s \gamma$ process, i.e. one would be able to discriminate among the SM and NP such as SUSY GUT models, the left-right symmetric standard model (LRSM), and the Pati-Salam models and so on, if one could precisely determine the chirality of the final $s$ quark. The $b$ quark can radiative decay into the $s$ quark in the $B$ meson and the chirality of the $s$ quark is almost determined as left-handed in the SM. Accordingly, if we find more right-handed $s$ quarks in the process than ones expected in the SM, we can expect that some kind of NP must cause this phenomena.

How about the measurement of the chirality of the $s$ quark in the $b \rightarrow s \gamma$ process for the NP search? One may naively think that the determination of chirality of quarks except for the top quark is impossible (the top quark can decay before the hadronization). The $b \rightarrow s \gamma$ process occurs through the dipole type operators, $\bar{s}_{L} \sigma_{\mu \nu} b F^{\mu \nu}\left(\bar{s}_{R} \sigma_{\mu \nu} b F^{\mu \nu}\right)$ which induce left- ( right- ) handed photon. The information on the chirality of the $s$ quark is imprinted on the photon polarization. In addition, there is no parity violation in QCD, the relation between the chirality of the $s$ quark and the photon polarization is unchanged even if the hadronization is taken into account. Therefore, one can determine the chirality of $s$ quark in the $b \rightarrow s \gamma$ process from the measurement of photon polarization $[4,5]$. In refs. $[6,7]$, the authors mentioned that the higher order correction may induce the right-handed photon even though it is in the SM.

At $e^{+} e^{-}$colliders, such as Belle and BaBar, $B_{d}$ mesons, which are spin 0 particles, are produced from the $\Upsilon(4 S)$ resonance. The photon polarization of the $B_{d} \rightarrow X_{s} \gamma$ decay is determined from measurements of hadronic angular distributions due to the conservation of angular momentum. The $\mathrm{LHCb}$ collaboration actually reported the result of observation of photon polarization by measuring the angular distribution of produced mesons in the $B \rightarrow K \pi \pi \gamma$ process [8]. ${ }^{1}$ In addition to the $B \rightarrow K \pi \pi \gamma$ process, there is another possibility to determine the photon polarization by the $B \rightarrow K^{*} l^{+} l^{-}$process. Although there exists a box diagram in the process, the radiative decay diagram (penguin) becomes dominant (the box diagram is suppressed) in a low invariant mass region of dileptons $[9,10]$ (see also [11]). The chirality of the $s$ quark in the $K^{*}$ meson can be lead by the chirality of photon due to the conservation of the spin. Then, it is important to discuss the possibility of the detection of the photon chirality. In this work, we will consider the $b \rightarrow s \gamma$ process. In particular, a ratio of the Wilson coefficients of a dipole operator and a polarization parameter of photon will be firstly evaluated at a typical point in a model of SUSY SU(5) GUT with the right-handed neutrino $\left(N_{R}\right)$ and LRSM in order to clarify whether one can find an evidence of NP or distinguish among the SM and NP, or not.

\footnotetext{
${ }^{1}$ This issue tells us that the theorists have to clarify the prediction of photon polarization in each model.
} 
In addition to the determination of the photon chirality, the $\mathrm{CP}$ asymmetry in the $b \rightarrow s \gamma$ process which are direct $\mathrm{CP}$ asymmetry, $A_{\mathrm{CP}}(b \rightarrow s \gamma)$ and time-dependent $\mathrm{CP}$ asymmetry, $S_{\mathrm{CP}}\left(B \rightarrow K_{s} \pi^{0} \gamma\right)$, is also a sensitive observable to NP [12]. Actually, the $\mathrm{CP}$ violating effects from NP can be enough larger than the $\mathrm{SM}$ expectation as $A_{\mathrm{CP}}(b \rightarrow$ $s \gamma) \simeq-0.5 \%$. However, this $\mathrm{CP}$ violation is constrained by the other experiment, e.g. the chromo electric dipole moment (CEDM) [13], and it can be negligibly smaller than the SM one when the $\mathrm{CP}$ violating phase depending on the $b \rightarrow s \gamma$ process is accidentally small. We will also evaluate the magnitude of $A_{\mathrm{CP}}(b \rightarrow s \gamma)$ in the SUSY SU(5) GUT with $N_{R}$ and the LRSM although the magnitude strongly depends on CP violating phases in the models. Furthermore, $S_{\mathrm{CP}}\left(B \rightarrow K_{s} \pi^{0} \gamma\right)$ can also become larger than the SM expectation as $S_{\mathrm{CP}}\left(B \rightarrow K_{s} \pi^{0} \gamma\right) \simeq-0.3$. We will show this value is insensitive to the $\mathrm{CP}$ phase. Then, we will compare experimental detectability for the models of NP between the determination of photon polarization and the observation of both CP asymmetry in the $b \rightarrow s \gamma$ process.

We will suggest that one can discriminate NP beyond the SM by the detection of photon polarization in $b \rightarrow s \gamma$ process. We will point out that time-dependent $\mathrm{CP}$ asymmetry is the most stringent constraint in our sample model point at the moment. However, it will actually turn out that the LHCb with $2 \mathrm{fb}^{-1}$ for the determination of photon polarization may check the existence of NP scale up to several $\mathrm{TeV}$ in both models.

\section{Photon polarization}

We investigate the photon polarization in the radiative rare decay, $b \rightarrow s \gamma$, process in a SUSY SU(5) GUT with $N_{R}$ and the LRSM for the search of NP. The Wilson coefficients $C_{7}$ and $C_{7}^{\prime}$ of the dipole operator for the $b \rightarrow s \gamma$ process are important for the analyses of photon polarization. The effective Hamiltonian reads

$$
\mathcal{H}_{\mathrm{eff}} \supset-\frac{4 G_{F}}{\sqrt{2}} V_{t b} V_{t s}^{*}\left(C_{7} O_{7}+C_{7}^{\prime} O_{7}^{\prime}\right)
$$

with the magnetic operator,

$$
O_{7}=\frac{e}{16 \pi^{2}} m_{b}\left(\bar{s} \sigma^{\mu \nu} P_{R} b\right) F_{\mu \nu},
$$

where $G_{F}$ is the Fermi constant, $m_{b}$ is the bottom quark mass, $\sigma^{\mu \nu}=\frac{i}{2}\left[\gamma^{\mu}, \gamma^{\nu}\right]$, and $P_{R, L}=\frac{1}{2}\left(1 \pm \gamma_{5}\right)$ (e.g., see [14]). $O_{7}^{\prime}$ is obtained by replacing $L \leftrightarrow R$ in $O_{7}$. Because left-handed $s$ quark comes from $O_{7}$ and right-handed one comes from $O_{7}^{\prime}$, we might be able to determine the chirality of $s$ quark by the difference of Wilson coefficients.

When one considers physics beyond the SM, there might be additional contributions to $C_{7}$ and $C_{7}^{\prime}$ from NP. In these cases, we can generically describe $C_{7}$ and $C_{7}^{\prime}$ as $C_{7}=$ $C_{7}^{\mathrm{SM}}+C_{7}^{\mathrm{NP}}$ and $C_{7}^{\prime}=C_{7}^{\prime \mathrm{SM}}+C_{7}^{\prime \mathrm{NP}}$, respectively. The coefficients at the $b$ quark mass scale $\mu_{b}$ are given by the leading logarithmic calculations with QCD corrections to the $b \rightarrow s \gamma$ process,

$$
\begin{aligned}
& C_{7}\left(\mu_{b}\right)=\eta^{\frac{16}{23}} C_{7}\left(m_{W}\right)+\frac{8}{3}\left(\eta^{\frac{14}{23}}-\eta^{\frac{16}{23}}\right) C_{8}\left(m_{W}\right)+\sum_{i=1}^{8} h_{i} \eta^{a_{i}} \\
& C_{7}^{\prime}\left(\mu_{b}\right)=\eta^{\frac{16}{23}} C_{7}^{\prime}\left(m_{W}\right)+\frac{8}{3}\left(\eta^{\frac{14}{23}}-\eta^{\frac{16}{23}}\right) C_{8}^{\prime}\left(m_{W}\right)
\end{aligned}
$$


at the leading order where $\eta=\alpha_{s}\left(m_{W}\right) / \alpha_{s}\left(\mu_{b}\right), \alpha_{s} \equiv g_{s}^{2} /(4 \pi), g_{s}$ is the strong coupling constant, $m_{W}$ is the $W$ boson mass, and $h_{i}$ and $a_{i}$ are numerical coefficients [15-17]. $C_{8}$ is the coefficient of chromomagnetic operator

$$
O_{8}=\frac{g_{s}}{16 \pi^{2}} m_{b}\left(\bar{s} \sigma^{\mu \nu} T^{A} P_{R} b\right) G_{\mu \nu}^{A},
$$

in the $\Delta F=1$ effective Hamiltonian,

$$
\mathcal{H}_{\text {eff }} \supset-\frac{4 G_{F}}{\sqrt{2}} V_{t b} V_{t s}^{*}\left(C_{8} O_{8}+C_{8}^{\prime} O_{8}^{\prime}\right)
$$

where $T^{A}$ are the generators of $\mathrm{SU}(3)_{C}$ and $O_{8}^{\prime}$ is also obtained by replacing $L \leftrightarrow R$ in $O_{8}$. And, we also describe $C_{8}=C_{8}^{\mathrm{SM}}+C_{8}^{\mathrm{NP}}$ and $C_{8}^{\prime}=C_{8}^{\prime \mathrm{SM}}+C_{8}^{\prime \mathrm{NP}}$ including contributions from NP.

\subsection{Case of SUSY SU(5) with the right-handed neutrinos}

\subsubsection{Model}

At first, we give a brief review of a model of SUSY SU(5) GUT with $N_{R}$. In a simple SU(5) GUT model, the final $s$ quark must have the same chirality as in the SM. When there is $N_{R}$, a neutrino Yukawa coupling induces additional flavor mixings in the right-handed down squark which derives the opposite chirality of $s$ quark. Thus, we adopt the model of SUSY SU(5) GUT with $N_{R}$. The superpotential in this model is given by

$$
W=\frac{1}{4} f_{i j}^{u} \Psi_{i} \Psi_{j} H+\sqrt{2} f_{i j}^{d} \Psi_{i} \Phi_{j} \bar{H}+f_{i j}^{\nu} \Phi_{i} \bar{N}_{j} H+M_{i j} \bar{N}_{i} \bar{N}_{j}
$$

where $\Psi_{i}$ are $\mathbf{1 0}$-dimensional multiplets, $\Phi_{i}$ are $\mathbf{5}$-dimensional ones, $N_{i}$ denote the righthanded neutrino superfields, and $H(\bar{H})$ is $\mathbf{5}$ - $(\overline{\mathbf{5}}-)$ dimensional Higgs multiplets. $i$ and $j$ mean the generation of the fermions, $i, j=1,2,3 . f^{u}, f^{d}$, and $f^{\nu}$ are Yukawa coupling matrices for the up-type quarks, down-type quarks (charged leptons) and neutrinos, respectively. These are given by

$$
\begin{aligned}
f_{i j}^{u} & =V_{k i} f_{u_{k}} e^{i \varphi_{u_{k}}} V_{k j}, \\
f_{i j}^{d} & =f_{d_{i}} \delta_{i j}, \\
f_{i j}^{\nu} & =e^{i \varphi_{d_{i}}} U_{i j}^{*} f_{\nu_{j}},
\end{aligned}
$$

without a loss of generality, where $V$ and $U$ are the Cabibbo-Kobayashi-Maskawa (CKM) and Pontecorvo-Maki-Nakagawa-Sakata (PMNS) matrices, respectively. $\varphi_{u_{k}}$ and $\varphi_{d_{i}}$ are CP-violating phases, and $f_{u_{k}}$ and $f_{d_{i}}$ are Yukawa couplings of the up- and down-type quarks (charged leptons), respectively. For the neutrinos sector, the light neutrino masses are given by the seesaw mechanism $m_{\nu_{i}}=f_{\nu_{i}}^{2} v_{u}^{2} / M_{N_{i}}$, where $v_{u}$ is the vacuum expectation value (VEV) of the up-type Higgs in $H$, and $M_{N_{i}}$ are the mass eigenvalues of the righthanded neutrinos. Here, we assume a diagonal right-handed Majorana mass matrix $M_{i j}$ for simplicity. 


\subsubsection{Photon polarization in SUSY SU(5) with right-handed neutrino}

We discuss $C_{7}$ and $C_{7}^{\prime}$, which determine the magnitude of the photon polarization, in the model of SUSY SU(5) GUT with $N_{R}$. In supersymmetric models, the dominant contributions to $C_{7,8}$ and $C_{7,8}^{\prime}$ arise from loop diagrams of the charged Higgses, charginos, and gluinos. Thus, $C_{7,8}^{\mathrm{NP}}=C_{7,8}^{H^{ \pm}}+C_{7,8}^{\tilde{\chi}^{ \pm}}+C_{7,8}^{\tilde{g}}$ and $C_{7,8}^{\prime \mathrm{NP}}=C_{7,8}^{\prime H^{ \pm}}+C_{7,8}^{\prime \tilde{\chi}^{ \pm}}+C_{7,8}^{\prime \tilde{g}}$ in the SUSY $\mathrm{SU}(5)$ model with $N_{R}$ where $C_{7,8}^{H^{ \pm}}\left(C_{7,8}^{\prime H^{ \pm}}\right), C_{7,8}^{\tilde{\chi}^{ \pm}}\left(C_{7,8}^{\prime \tilde{\chi}^{ \pm}}\right)$, and $C_{7,8}^{\tilde{g}}\left(C_{7,8}^{\prime \tilde{g}}\right)$ are the contributions to $C_{7,8}\left(C_{7,8}^{\prime}\right)$ from the charged Higgses, charginos, and gluinos, respectively. These contributions are calculated as [18]

$$
\begin{aligned}
C_{7}^{H^{ \pm}} & =C_{7}^{\prime H^{ \pm}} \simeq\left(\frac{1-\epsilon t_{\beta}}{1+\epsilon t_{\beta}}\right) \frac{1}{2} h_{7}\left(y_{t}\right), \\
C_{7}^{\tilde{\chi}^{ \pm}} & =\frac{4 G_{F}}{\sqrt{2}} \frac{g_{2}^{2}}{\tilde{m}^{2}}\left[\frac{\left(\delta_{u}^{L L}\right)_{32}}{V_{t b} V_{t s}^{*}} \frac{\mu M_{2}}{\tilde{m}^{2}} f_{7}^{(1)}\left(x_{2}, x_{\mu}\right)+\frac{m_{t}^{2}}{M_{W}^{2}} \frac{A_{t} \mu}{\tilde{m}^{2}} f_{7}^{(2)}\left(x_{\mu}\right)\right] \frac{t_{\beta}}{1+\epsilon t_{\beta}}, \\
C_{7}^{\prime} \tilde{\chi}^{ \pm} & =\frac{4 G_{F}}{\sqrt{2}} \frac{g_{2}^{2}}{\tilde{m}^{2}}\left[\frac{\left(\delta_{u}^{R R}\right)_{32}}{V_{t b} V_{t s}^{*}} \frac{\mu M_{2}}{\tilde{m}^{2}} f_{7}^{(1)}\left(x_{2}, x_{\mu}\right)+\frac{m_{t}^{2}}{M_{W}^{2}} \frac{A_{t} \mu}{\tilde{m}^{2}} f_{7}^{(2)}\left(x_{\mu}\right)\right] \frac{t_{\beta}}{1+\epsilon t_{\beta}}, \\
C_{7}^{\tilde{g}^{ \pm}} & =\frac{4 G_{F}}{\sqrt{2}} \frac{g_{s}^{2}}{\tilde{m}^{2}}\left[\frac{M_{\tilde{g}}}{m_{b}} \frac{\left(\delta_{d}^{R L}\right)_{32}}{V_{t b} V_{t s}^{*}} g_{7}^{(1)}\left(x_{g}\right)+\frac{M_{\tilde{g}} \mu}{\tilde{m}^{2}} \frac{t_{\beta}}{1+\epsilon t_{\beta}} \frac{\left(\delta_{d}^{L L}\right)_{32}}{V_{t b} V_{t s}^{*}} g_{7}^{(2)}\left(x_{g}\right)\right], \\
C_{7}^{\prime} \tilde{g}^{ \pm} & =\frac{4 G_{F}}{\sqrt{2}} \frac{g_{s}^{2}}{\tilde{m}^{2}}\left[\frac{M_{\tilde{g}}}{m_{b}} \frac{\left(\delta_{d}^{L R}\right)_{32}}{V_{t b} V_{t s}^{*}} g_{7}^{(2)}\left(x_{g}\right)+\frac{M_{\tilde{g}} \mu^{*}}{\tilde{m}^{2}} \frac{t_{\beta}}{1+\epsilon t_{\beta}} \frac{\left(\delta_{d}^{R R}\right)_{32}}{V_{t b} V_{t s}^{*}} g_{7}^{(1)}\left(x_{g}\right)\right],
\end{aligned}
$$

and

$$
\begin{aligned}
C_{8}^{H^{ \pm}} & =C_{8}^{\prime H^{ \pm}} \simeq\left(\frac{1-\epsilon t_{\beta}}{1+\epsilon t_{\beta}}\right) \frac{1}{2} h_{8}\left(y_{t}\right), \\
C_{8}^{\tilde{\chi}^{ \pm}} & =\frac{4 G_{F}}{\sqrt{2}} \frac{g_{2}^{2}}{\tilde{m}^{2}}\left[\frac{\left(\delta_{u}^{L L}\right)_{32}}{V_{t b} V_{t s}^{*}} \frac{\mu M_{2}}{\tilde{m}^{2}} f_{8}^{(1)}\left(x_{2}, x_{\mu}\right)+\frac{m_{t}^{2}}{M_{W}^{2}} \frac{A_{t} \mu}{\tilde{m}^{2}} f_{8}^{(2)}\left(x_{\mu}\right)\right] \frac{t_{\beta}}{1+\epsilon t_{\beta}}, \\
C_{8}^{\prime \tilde{\chi}^{ \pm}} & =\frac{4 G_{F}}{\sqrt{2}} \frac{g_{2}^{2}}{\tilde{m}^{2}}\left[\frac{\left(\delta_{u}^{R R}\right)_{32}}{V_{t b} V_{t s}^{*}} \frac{\mu M_{2}}{\tilde{m}^{2}} f_{8}^{(1)}\left(x_{2}, x_{\mu}\right)+\frac{m_{t}^{2}}{M_{W}^{2}} \frac{A_{t} \mu}{\tilde{m}^{2}} f_{8}^{(2)}\left(x_{\mu}\right)\right] \frac{t_{\beta}}{1+\epsilon t_{\beta}}, \\
C_{8}^{\tilde{g}^{ \pm}} & =\frac{4 G_{F}}{\sqrt{2}} \frac{g_{s}^{2}}{\tilde{m}^{2}}\left[\frac{M_{\tilde{g}}}{m_{b}} \frac{\left(\delta_{d}^{R L}\right)_{32}}{V_{t b} V_{t s}^{*}} g_{8}^{(1)}\left(x_{g}\right)+\frac{M_{\tilde{g}} \mu}{\tilde{m}^{2}} \frac{t_{\beta}}{1+\epsilon t_{\beta}} \frac{\left(\delta_{d}^{L L}\right)_{32}}{V_{t b} V_{t s}^{*}} g_{8}^{(2)}\left(x_{g}\right)\right], \\
C_{8}^{\prime} \tilde{g}^{ \pm} & =\frac{4 G_{F}}{\sqrt{2}} \frac{g_{s}^{2}}{\tilde{m}^{2}}\left[\frac{M_{\tilde{g}}}{m_{b}} \frac{\left(\delta_{d}^{L R}\right)_{32}}{V_{t b} V_{t s}^{*}} g_{8}^{(2)}\left(x_{g}\right)+\frac{M_{\tilde{g}} \mu^{*}}{\tilde{m}^{2}} \frac{t_{\beta}}{1+\epsilon t_{\beta}} \frac{\left(\delta_{d}^{R R}\right)_{32}}{V_{t b} V_{t s}^{*}} g_{8}^{(1)}\left(x_{g}\right)\right],
\end{aligned}
$$

at the weak scale and $\epsilon \simeq \alpha_{s} /(3 \pi) \sim \mathcal{O}\left(10^{-2}\right)$ for a degenerate SUSY spectrum, $t_{\beta}=$ $\tan \beta \equiv v_{u} / v_{d}, v_{d}$ is the VEV of down-type Higgs, $g_{2}$ is the $\mathrm{SU}(2)_{L}$ gauge coupling constant, $\tilde{m}$ is an averaged squark mass, $\left(\delta_{q}^{X Y}\right)_{i j}(q=u, d$ and $X, Y=L, R)$ are mass insertion parameters, $\mu$ is the supersymmetric Higgs mass, $M_{x}(x=2, \tilde{g})$ are the gaugino masses, and $A_{t}$ is the soft scalars coupling for the top quark. $h_{7,8}, f_{7,8}^{(1,2)}$, and $g_{7,8}^{(1,2)}$ are loop functions, which are given in appendix A. The mass insertion parameters are given in appendix B. And, we define $y_{t} \equiv m_{t}^{2} / M_{H^{ \pm}}^{2}, x_{2} \equiv\left|M_{2}\right|^{2} / \tilde{m}^{2}, x_{\mu} \equiv|\mu|^{2} / \tilde{m}^{2}$, and $x_{g} \equiv M_{\tilde{g}}^{2} / \tilde{m}^{2}$ where $m_{t}$ and $M_{H^{ \pm}}$are the top quark and charged Higgs masses, respectively. The contributions from the charged Higgs and chargino to $C_{7}^{\prime N P}$ are suppressed by $m_{s} / m_{b}$.

In order to see the magnitude of contributions from $\mathrm{NP}$, we estimate the ratio $\left|C_{7}^{\prime} / C_{7}\right|$ at the $b$ quark mass scale, which determines the size of polarization of photon as seen 


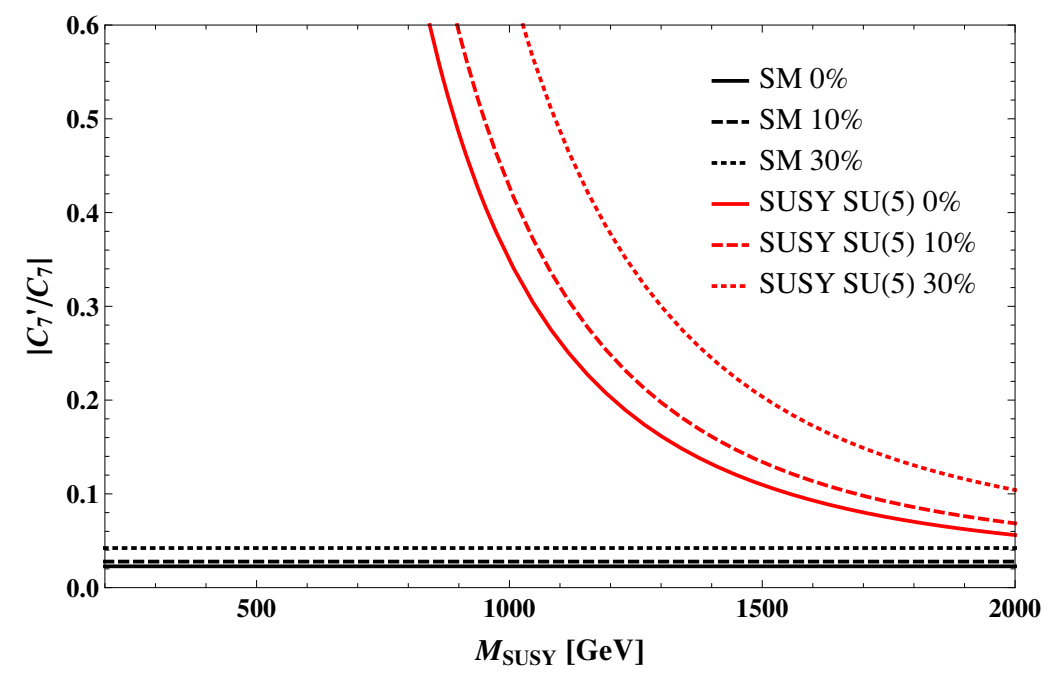

Figure 1. The magnitude of $\left|C_{7}^{\prime} / C_{7}\right|$ in the SM and SUSY SU(5) GUT with $N_{R}$ which are depicted by the black and red curves, respectively. The contours with $n \%$ correspond to cases that there is $n \%$ misidentification in the $C_{7}$ measurement at experiments, i.e. each of the contours denotes $\left|C_{7}^{\prime}(1+n / 100) /\left(C_{7}(1-n / 100)\right)\right|$.

below. The value of the ratio in the model of SUSY SU(5) GUT with $N_{R}$ is shown by the red solid curve in figure 1. The black solid line indicates the SM case $\left|C_{7}^{\prime \mathrm{SM}} / C_{7}^{\mathrm{SM}}\right|$ which can be approximated as $\left|C_{7}^{\prime \mathrm{SM}} / C_{7}^{\mathrm{SM}}\right| \simeq m_{s} / m_{b}$. One can see that $\left|C_{7}^{\prime} / C_{7}\right|$ in the SUSY $\mathrm{SU}(5)$ with $N_{R}$ case is enhanced from the SM. This means that the final state of $s_{R}$ in $b \rightarrow s \gamma$ increases compared to the SM while the most of final state of $b \rightarrow s \gamma$ in the SM is $s_{L}$ due to the suppression proportional to $m_{s} / m_{b}$.

In figure 1, the horizontal axis is a typical scale of NP, which is a SUSY breaking scale $M_{\mathrm{SUSY}}$ in the SUSY SU(5) GUT with $N_{R}$ case. One can see that at a large limit of $M_{\mathrm{SUSY}}$, the ratio $\left|C_{7}^{\prime} / C_{7}\right|$ closes to the $\mathrm{SM}$ case, $\left|C_{7}^{\prime} / C_{7}\right| \rightarrow\left|C_{7}^{\prime \mathrm{SM}} / C_{7}^{\mathrm{SM}}\right|$. The contours with $n \%$ correspond to case that there is $n \%$ misidentification in the $C_{7}$ measurement at experiments, i.e. the contours denote $\left|C_{7}^{\prime}(1+n / 100) /\left(C_{7}(1-n / 100)\right)\right|$. This misidentification corresponds to a mismatch in the conversion of left-handed helicity to the left-handed chirality. (The helicity is determined in experiments.) For instance, if one identifies the left-handed helicity with the left-handed chirality with $10 \%$ misidentification, the right-handed chirality is over estimated as $110 \%$ of the true value. Thus, the contours go above as $n$ increases in figure 1 . In the calculation, we take

$$
\begin{aligned}
& t_{\beta}=10, \quad M_{\mathrm{SUSY}}=m_{1 / 2}, \quad m_{0}=A_{0}=A_{t}=A_{b}=1 \mathrm{TeV}, \\
& \mu=1.01 m_{0}, \quad M_{2}=0.822 M_{\mathrm{SUSY}}, \quad M_{\tilde{g}}=2.86 M_{\mathrm{SUSY}}, \\
& M_{H_{c}}=10^{16} \mathrm{GeV}, \quad \quad \tilde{m}_{\tilde{u}}^{2}=\sqrt{m_{\tilde{Q}_{L}}^{2} m_{\tilde{u}_{R}}^{2}}, \quad \tilde{m}_{\tilde{d}}^{2}=\sqrt{m_{\tilde{Q}_{L}}^{2} m_{\tilde{d}_{R}}^{2}}, \\
& m_{\tilde{Q}_{L}}^{2}=m_{0}^{2}+6.86 M_{\mathrm{SUSY}}^{2}, \quad m_{\tilde{u}_{R}}^{2}=m_{0}^{2}+6.44 M_{\mathrm{SUSY}}^{2}, \quad m_{\tilde{d}_{R}}^{2}=m_{0}^{2}+6.39 M_{\mathrm{SUSY}}^{2}, \\
& f_{\nu_{i}}=1, \quad \varphi_{u_{23}}=0.01, \quad \varphi_{d_{23}}=\pi,
\end{aligned}
$$




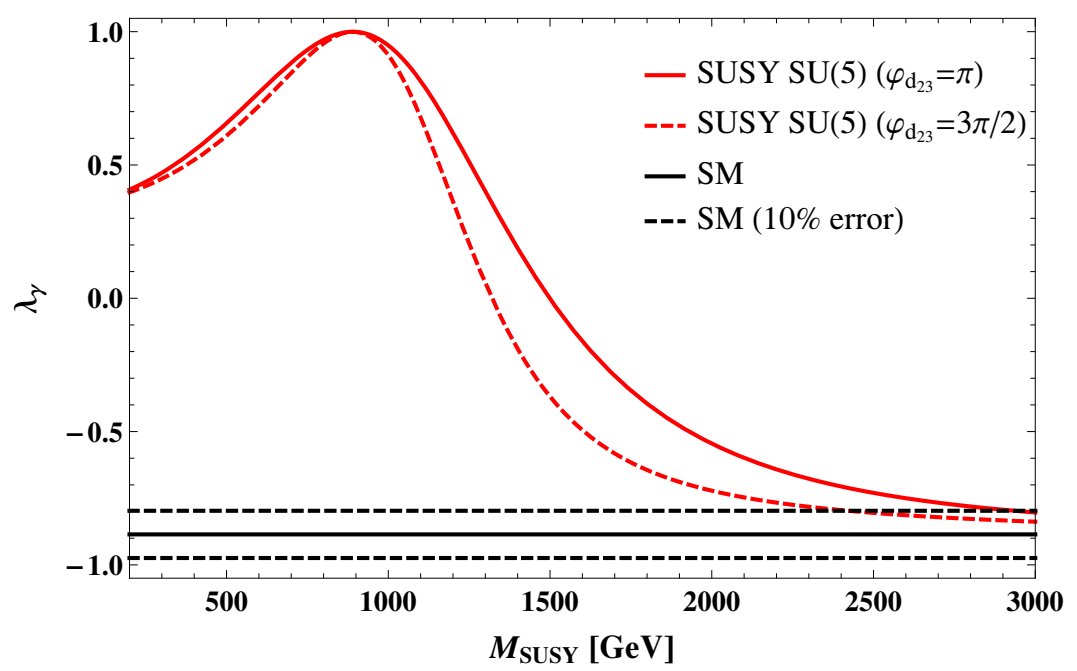

Figure 2. The polarization parameter $\lambda_{\gamma}$ in $b \rightarrow s \gamma$ in the SM and SUSY SU(5) GUT with $N_{R}$ which are depicted by the black and red curves, respectively. We also show $\varphi_{d_{23}}=3 \pi / 2$ case by the red dashed curve and $10 \%$ error in the SM prediction by the black dashed curve for comparison.

as a typical point where $\mathrm{CP}$ phases are given in the radian unit. ${ }^{2}$ We take other values of parameters in the SM and the neutrino sector (PMNS mixing angles) as the best fit values given in $[19,20]$.

Next, we consider the polarization parameter of photon $\lambda_{\gamma}$ at the $b$ quark mass scale defined as

$$
\lambda_{\gamma} \equiv \frac{\operatorname{Re}\left[C_{7}^{\prime} / C_{7}\right]^{2}+\operatorname{Im}\left[C_{7}^{\prime} / C_{7}\right]^{2}-1}{\operatorname{Re}\left[C_{7}^{\prime} / C_{7}\right]^{2}+\operatorname{Im}\left[C_{7}^{\prime} / C_{7}\right]^{2}+1}
$$

In order to measure $\lambda_{\gamma}$ we need to consider a parity-odd observable in the $B_{d} \rightarrow X_{s} \gamma$ decay since the photon polarization is parity-odd. In refs. $[4,5]$ they proposed that $\lambda_{\gamma}$ can be measured from the up-down asymmetry of the photon direction relative to the $K \pi \pi$ decay plane in the $K_{1}(1400)$ rest frame. We show $\lambda_{\gamma}$ in figure 2 and the SM and SUSY SU(5) GUT with $N_{R}$ cases are depicted by the black and red curves, respectively. We also show $\varphi_{d_{23}}=3 \pi / 2$ case by the red dashed curve for comparison. The SM prediction for the photon polarization still have roughly $10 \%$ error due to $\mathcal{O}_{2}$ operator [11], then we depict it by the black dashed curve.

The superKEKB with an integrated luminosity of $75 \mathrm{ab}^{-1}$ and the LHCb with $2 \mathrm{fb}^{-1}$ might reach the $20\left(\left|\lambda_{\gamma}\right|=0.8\left|\lambda_{\gamma}^{\mathrm{SM}}\right|\right)$ and $10 \%\left(\left|\lambda_{\gamma}\right|=0.9\left|\lambda_{\gamma}^{\mathrm{SM}}\right|\right)$ precision, respectively $[11,21]$. Thus, the future experiment will be able to check the NP scale up to about $1700 \mathrm{GeV}$ (which corresponds to $M_{2} \simeq 1400 \mathrm{GeV}$ and $M_{\tilde{g}} \simeq 4900 \mathrm{GeV}$ ) in this model of SUSY SU(5) with $N_{R}$.

\footnotetext{
${ }^{2}$ Note that $\varphi_{d_{23}}$ is sensitive to the CEDM. The allowed minimal and maximal values by the CEDM constraint are $\pi$ and $3 \pi / 2$ (or $\pi / 2$ ), respectively. In the calculation of photon polarization, we take the minimal value. The photon polarization is not so sensitive to the value of $\varphi_{d_{23}}$ but the difference between these values appears in the calculation of direct CP asymmetry as we will show in section 3 . We have numerically checked that another phase, $\varphi_{u_{23}}$, is not sensitive to our evaluation.
} 


\subsection{Case of Left-right symmetric standard model}

\subsubsection{Model}

Next, we consider the case in the left-right symmetric standard model (LRSM) [22-25]. The model is based on the gauge group $\mathrm{SU}(2)_{L} \times \mathrm{SU}(2)_{R} \times \mathrm{U}(1)_{\tilde{Y}}$. In the model, the SM left-handed doublet fermions are $\mathrm{SU}(2)_{R}$ singlets, and the right-handed fermions including the neutrinos are $\mathrm{SU}(2)_{R}$ doublets and $\mathrm{SU}(2)_{L}$ singlets. For the Higgs sector, the model includes a bi-doublet scalar $\Phi$ under the $\mathrm{SU}(2)_{L} \times \mathrm{SU}(2)_{R}$ transformation, an $\mathrm{SU}(2)_{R}$ triplet $\Delta_{R}$, and an $\mathrm{SU}(2)_{L}$ triplet $\Delta_{L}$ in order to realize a realistic symmetry breaking, $\mathrm{SU}(2)_{L} \times \mathrm{SU}(2)_{R} \times \mathrm{U}(1)_{\tilde{Y}} \rightarrow \mathrm{SU}(2)_{L} \times \mathrm{U}(1)_{Y} \rightarrow \mathrm{U}(1)_{\mathrm{em}}$. The symmetry breaking can be undertaken by the VEVs of $\Phi, \Delta_{R}$, and $\Delta_{L}$ as

$$
\langle\Phi\rangle=\left(\begin{array}{cc}
\kappa & 0 \\
0 & \kappa^{\prime} e^{i \omega}
\end{array}\right), \quad\left\langle\Delta_{R}\right\rangle=\left(\begin{array}{cc}
0 & 0 \\
v_{R} & 0
\end{array}\right), \quad\left\langle\Delta_{L}\right\rangle=\left(\begin{array}{cc}
0 & 0 \\
v_{L} e^{i \theta_{L}} & 0
\end{array}\right),
$$

with six real numbers $\kappa, \kappa^{\prime}, \omega, v_{R}, v_{L}$, and $\theta_{L}$. Regarding the magnitude of the VEVs, $v_{R}$ should be much larger than the electroweak (EW) scale to suppress the right-handed currents at low energy, and the EW $\rho$-parameter limits $v_{L}$ to be $v_{L} \lesssim 10 \mathrm{GeV}$ [26]. In this work, we take $v_{L}=0$ for simplicity, which is usually taken in literatures (e.g., [27, $28]$ ). Since the VEV of $\Phi$ leads to the standard EW symmetry breaking, we define $v \equiv$ $\sqrt{\kappa^{2}+\kappa^{\prime 2}}=174 \mathrm{GeV}, \tan \beta_{\mathrm{LR}} \equiv \kappa / \kappa^{\prime}$, and $\epsilon_{\mathrm{LR}} \equiv v / v_{R}$.

The charged gauge bosons are given by the admixture of the mass eigenstates as

$$
\left(\begin{array}{l}
W_{L}^{-} \\
W_{R}^{-}
\end{array}\right)=\left(\begin{array}{cc}
\cos \zeta & -\sin \zeta e^{i \omega} \\
\sin \zeta e^{i \omega} & \cos \zeta
\end{array}\right)\left(\begin{array}{l}
W_{1}^{-} \\
W_{2}^{-}
\end{array}\right) .
$$

The masses of charged gauge bosons are approximated as

$$
M_{W_{1}} \simeq \frac{g_{L} v}{\sqrt{2}}\left(1-\epsilon_{\mathrm{LR}}^{2} \sin ^{2} \beta_{\mathrm{LR}} \cos ^{2} \beta_{\mathrm{LR}}\right), \quad M_{W_{2}} \simeq g_{R} v_{R}\left(1+\frac{1}{4} \epsilon_{\mathrm{LR}}^{2}\right),
$$

where $g_{L, R}$ are the gauge couplings of $\mathrm{SU}(2)_{L, R}$ and we take $g_{R} / g_{L}=1$ for simplicity in the numerical analysis. The mixing angle is given as $\sin \zeta \approx\left(M_{W_{1}}^{2} / M_{W_{2}}^{2}\right) \sin 2 \beta_{L R}$. $M_{W_{2}}$ is identified with $M_{\mathrm{NP}}$ in the LRSM. There are also charged and heavy neutral Higgs bosons in the LRSM. And their masses are nearly the same, $M_{H^{ \pm}} \simeq M_{H^{0}} \simeq M_{A^{0}}$, where $M_{H^{ \pm}}$are the masses of the charged Higgs bosons and $M_{H^{0}, A^{0}}$ are the neutral Higgs bosons masses [27, 29]. In this work, we represent both the charged and neutral Higgs bosons masses as $M_{H}$ for simplicity. Regarding the flavor mixing matrices, we assume $V=V_{L}=V_{R}$, where $V_{L}$ and $V_{R}$ are the mixing matrices for the left- and right-handed quarks, respectively. $V=V_{L}=V_{R}$ is taken in the so-called the manifest LRSM [30, 31] and we also take the equality in this work. In our numerical analyses, we have three free parameters, i.e. $M_{H}, M_{\mathrm{NP}}=M_{W_{2}}$, and $\tan \beta_{\mathrm{LR}} \cdot{ }^{3}$

\footnotetext{
${ }^{3}$ The value of $M_{W_{2}}$ is not exactly determined even if one takes $g_{R} / g_{L}=1$ and fixes the value of $M_{H}$, because the heavy Higgs masses depend on scalar quartic couplings, which can be in region from 0 to $4 \pi$, and/or trilinear couplings. Thus, one can generally take both $M_{W_{2}}$ and $M_{H}$ as free parameters in this model.
} 


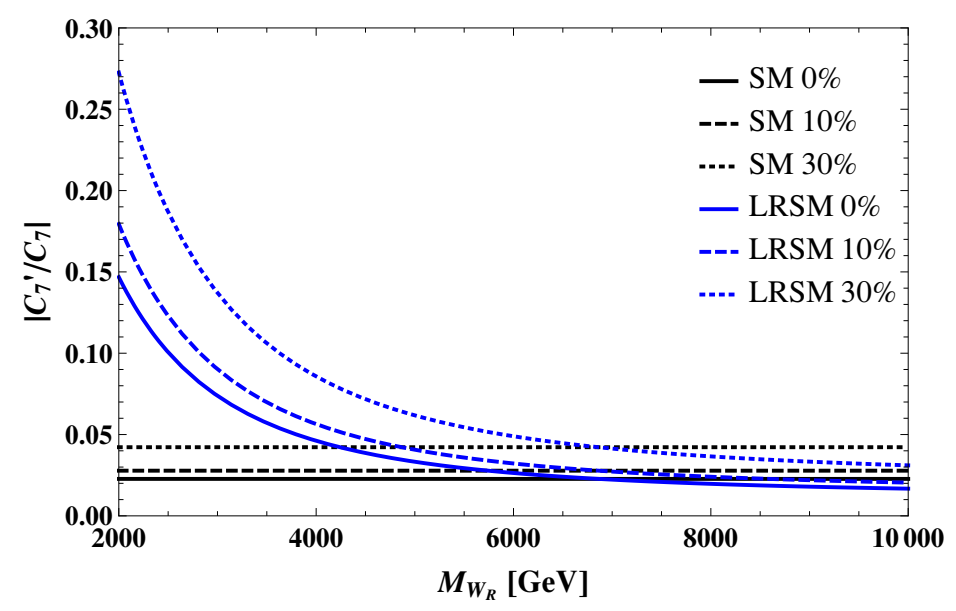

Figure 3. The magnitude of $\left|C_{7}^{\prime} / C_{7}\right|$ in the SM and LRSM which are depicted by the black and blue curves, respectively. The meaning of each contour is the same as figure 1.

\subsubsection{Photon polarization in LRSM}

$C_{7}^{\mathrm{NP}}$ and $C_{7}^{\prime \mathrm{NP}}$ in the LRSM are generally given by

$$
\begin{aligned}
C_{7}^{\mathrm{NP}}= & -\sin ^{2} \zeta\left(D_{0}^{\prime}\left(x_{t}\right)-\frac{M_{W_{1}}^{2}}{M_{W_{2}}^{2}} D_{0}^{\prime}\left(\tilde{x}_{t}\right)\right) \\
& +\frac{m_{t}}{m_{b}} \frac{g_{R}}{g_{L}} \frac{V_{t b}^{R}}{V_{t b}} \sin \zeta \cos \zeta e^{i \omega}\left(A_{\mathrm{LR}}\left(x_{t}\right)-\frac{M_{W_{1}}^{2}}{M_{W_{2}}^{2}} A_{\mathrm{LR}}\left(\tilde{x}_{t}\right)\right) \\
& +\frac{m_{c}}{m_{b}} \frac{g_{R}}{g_{L}} \frac{V_{c s}^{*} V_{c b}^{R}}{V_{t s}^{*} V_{t b}} \sin \zeta \cos \zeta e^{i \omega}\left(A_{\mathrm{LR}}\left(x_{c}\right)-\frac{M_{W_{1}}^{2}}{M_{W_{2}}^{2}} A_{\mathrm{LR}}\left(\tilde{x}_{c}\right)\right)+\frac{m_{t}}{m_{b}} \frac{\tan \left(2 \beta_{\mathrm{LR}}\right)}{\cos \left(2 \beta_{\mathrm{LR}}\right)} e^{i \omega} \frac{V_{t b}^{R}}{V_{t b}} h_{7}(y) \\
& +\tan \left(2 \beta_{\mathrm{LR}}\right) A_{H}^{2}(x), \\
C_{7}^{\prime \mathrm{NP}}= & \frac{g_{R}^{2}}{g_{L}^{2}} \frac{V_{t s}^{R *} V_{t b}^{R}}{V_{t s}^{*} V_{t b}}\left(\sin { }^{2} \zeta D_{0}^{\prime}\left(x_{t}\right)+\cos ^{2} \zeta \frac{M_{W_{1}}^{2}}{M_{W_{2}}^{2}} D_{0}^{\prime}\left(\tilde{x}_{t}\right)\right) \\
& +\frac{m_{t}}{m_{b}} \frac{g_{R}}{g_{L}} \frac{V_{t s}^{R *}}{V_{t s}^{*}} \sin \zeta \cos \zeta e^{-i \omega}\left(A_{\mathrm{LR}}\left(x_{t}\right)-\frac{M_{W_{1}}^{2}}{M_{W_{2}}^{2}} A_{\mathrm{LR}}\left(\tilde{x}_{t}\right)\right) \\
& +\frac{m_{c}}{m_{b}} \frac{g_{R}}{g_{L}} \frac{V_{c s}^{R *} V_{c b}}{V_{t s}^{*} V_{t b}} \sin \zeta \cos \zeta e^{-i \omega}\left(A_{\mathrm{LR}}\left(x_{c}\right)-\frac{M_{W_{1}}^{2}}{M_{W_{2}}^{2}} A_{\mathrm{LR}}\left(\tilde{x}_{c}\right)\right) \\
& +\frac{m_{t}}{m_{b}} \frac{\tan \left(2 \beta_{\mathrm{LR}}\right)}{\cos \left(2 \beta_{\mathrm{LR}}\right)} e^{-i \omega} \frac{V_{t s}^{R *}}{V_{t s}^{*}} h_{7}(y)+\frac{V_{t s}^{R *} V_{t b}^{R}}{V_{t s}^{*} V_{t b}} \frac{1}{\cos ^{2}\left(2 \beta_{\mathrm{LR}}\right)} A_{H}^{2}(x),
\end{aligned}
$$

when one does not assume $g_{R} / g_{L}=1$ and $V=V_{R}$, where loop functions $D_{0}^{\prime}(x)$ and $A_{\mathrm{LR}}(x)$ are given in appendix $\mathrm{A}$, and $x_{t} \equiv m_{t}^{2} / m_{W_{1}}^{2}, \tilde{x}_{t} \equiv m_{t}^{2} / m_{W_{2}}^{2}, x_{c} \equiv m_{c}^{2} / m_{W_{1}}^{2}, \tilde{x}_{c} \equiv m_{c}^{2} / m_{W_{2}}^{2}$, and $y \equiv m_{t}^{2} / M_{H}^{2}$. The ratio $\left|C_{7}^{\prime} / C_{7}\right|$ in the LRSM is shown by the blue curves in figure 3 . The value of the ratio in the LRSM is larger than the both cases of SM and SUSY SU(5) with $N_{R}$ model because the right-handed current in the LRSM is more effective than those 


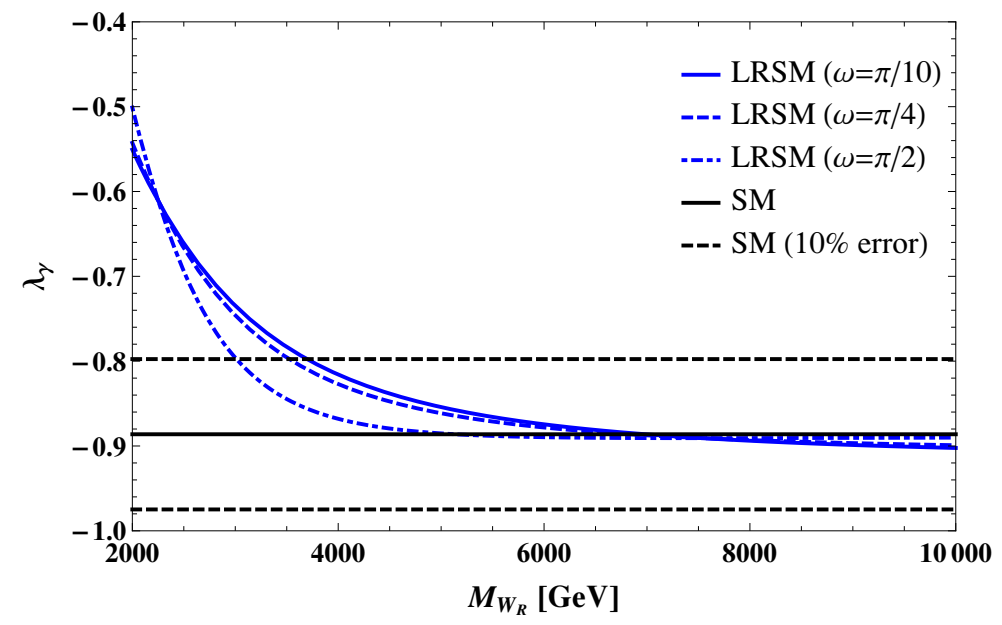

Figure 4. The polarization parameter $\lambda_{\gamma}$ in $b \rightarrow s \gamma$ in the SM and LRSM which are depicted by the black and blue curves, respectively. We also show $\omega=\pi / 4$ and $\pi / 2$ cases by the blue dashed and dashed-dotted curves for comparison, respectively.

models. In the analysis, we take

$$
\frac{g_{R}}{g_{L}}=1, \quad V=V^{R}, \quad \tan \beta_{\mathrm{LR}}=10, \quad \omega=\frac{\pi}{10}, \quad M_{H^{ \pm}}=15 \mathrm{TeV},
$$

as a sample point. ${ }^{4}$

The polarization parameter in the LRSM is shown by the blue curve in figure 4 . The SM and LRSM cases are depicted by the black and blue curves, respectively. We also show $\omega=\pi / 4$ and $\pi / 2$ cases by the blue dashed and dashed-dotted curves for comparison, respectively. Since $M_{\mathrm{NP}} \simeq 3.7 \mathrm{TeV}$ for $10 \%$ deviation from the SM prediction, the future $\mathrm{LHCb}$ experiment with $2 \mathrm{fb}^{-1}$ will check the scale of the right-handed gauge boson up to 3.7 TeV in this case. The dependence of photon polarization on the $\mathrm{CP}$ phase is not strong.

\section{CP asymmetry}

Next, we evaluate the $\mathrm{CP}$ asymmetry in $b \rightarrow s \gamma$ process in each model. The $\mathrm{CP}$ asymmetry can be categorized into two parts: one is the direct CP asymmetry which is induced by the $\mathrm{CP}$ phase in the decay amplitude, and the other is the time-dependent $\mathrm{CP}$ asymmetry which is induced during the meson mixing.

\subsection{Direct CP asymmetry}

In addition to the determination of photon polarization, the observation of $\mathrm{CP}$ asymmetry in the $b \rightarrow s \gamma$ process is still sensitive to the existence of NP. Thus, we evaluate the CP asymmetry of the process in both models of SUSY SU(5) GUT with $N_{R}$ and LRSM. The

\footnotetext{
${ }^{4}$ Although there are crossing points of the NP lines with the SM prediction line in all figures afterward, they are due to the fixing of the charged Higgs mass.
} 


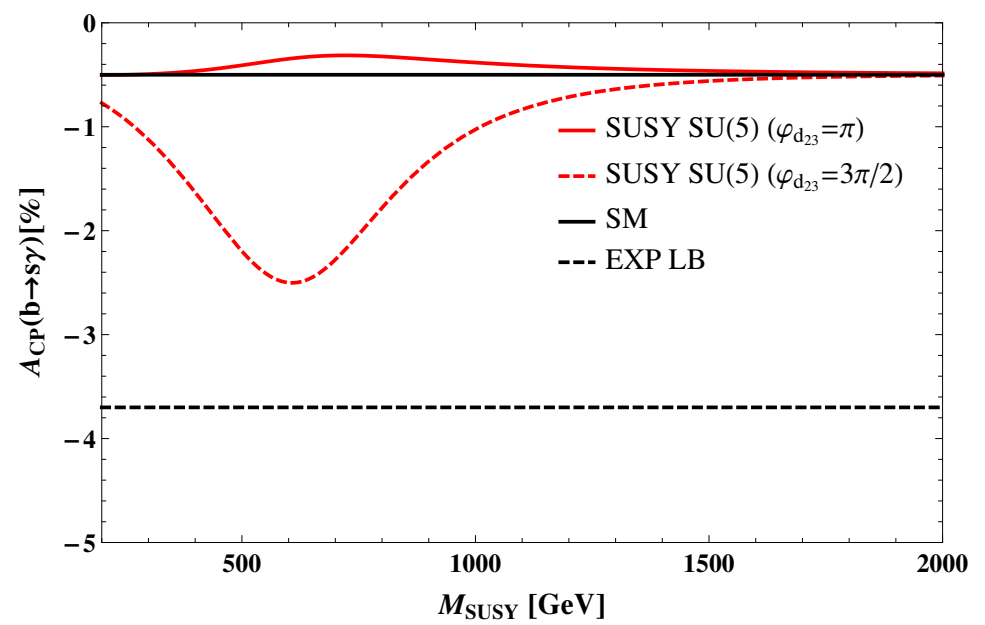

Figure 5. The direct CP asymmetry in $b \rightarrow s \gamma$ in the SM and SUSY SU(5) GUT with $N_{R}$, which are depicted by the black and red solid curves. The black dashed line corresponds to the current experimental lower bound [33]. We also show the $\varphi_{d_{23}}=3 \pi / 2$ case by the red dashed curve for comparison.

asymmetry is given by

$$
\begin{aligned}
A_{\mathrm{CP}}(b \rightarrow s \gamma) \equiv & \frac{\Gamma\left(B \rightarrow X_{\bar{s}} \gamma\right)-\Gamma\left(\bar{B} \rightarrow X_{s} \gamma\right)}{\Gamma\left(B \rightarrow X_{\bar{s}} \gamma\right)+\Gamma\left(\bar{B} \rightarrow X_{s} \gamma\right)} \\
\simeq & -\frac{1}{\left|C_{7}\right|^{2}+\left|C_{7}^{\prime}\right|^{2}}\left(1.23 \operatorname{Im}\left[C_{2} C_{7}^{*}\right]-9.52 \operatorname{Im}\left[C_{8} C_{7}^{*}+C_{8}^{\prime} C_{7}^{\prime *}\right]+0.10 \operatorname{Im}\left[C_{2} C_{8}^{*}\right]\right) \\
& -0.5 \quad(\text { in } \%),
\end{aligned}
$$

which of course strongly depends on the CP-phases in the model where $C_{2}$ is the coefficient of the operator $O_{2}=\left(\bar{c}^{\alpha} \gamma_{\mu} P_{L} b^{\alpha}\right)\left(\bar{c}^{\beta} \gamma^{\mu} P_{L} b^{\beta}\right)$ in the effective Hamiltonian of $\Delta F=2$ transitions. Note that the contributions from NP to $C_{2}$ and $C_{2}^{\prime}$ are negligibly small while $C_{7,8}$ and $C_{7,8}^{\prime}$ include contributions from NP, i.e. $C_{2}^{\prime} \ll C_{2}=C_{2}^{\mathrm{SM}}=1$. Thus, $A_{\mathrm{CP}}(b \rightarrow s \gamma)$ is well approximated by eq. $(3.1) .^{5}$

\subsubsection{SUSY SU(5) with right-handed neutrino case}

We show $A_{\mathrm{CP}}(b \rightarrow s \gamma)$ in the SUSY SU(5) with $N_{R}$ and SM cases by red and black solid curves in figure 5, respectively. The experimental lower bound as $-3.7 \%$ [19] is also shown by the black dashed line. We also show $\varphi_{d_{23}}=3 \pi / 2$ case by the red dashed curve for comparison. It turns out that the magnitude of the direct $\mathrm{CP}$ asymmetry in the case of SUSY SU(5) with $N_{R}$ highly depend on the phase $\varphi_{d_{23}}$. The current experimental lower bound does not constrain the scale of NP, $M_{\mathrm{SUSY}}$ in this parameter setup. Therefore, the measurement of $A_{\mathrm{CP}}$ does not currently constrain on the NP scale in the SUSY SU(5) with $N_{R}$ model even if one takes the maximally allowed CP phase as $\varphi_{d_{23}}=3 \pi / 2$. But, the expected reach of Belle II with $50 \mathrm{ab}^{-1}$ for $A_{\mathrm{CP}}\left(B \rightarrow X_{s+d} \gamma\right)$ will be $\pm 2 \%$ precision. Thus,

\footnotetext{
${ }^{5}$ There is also an error in the SM prediction but it is enough small [32]. Therefore, we neglect such correction here just for simplicity.
} 


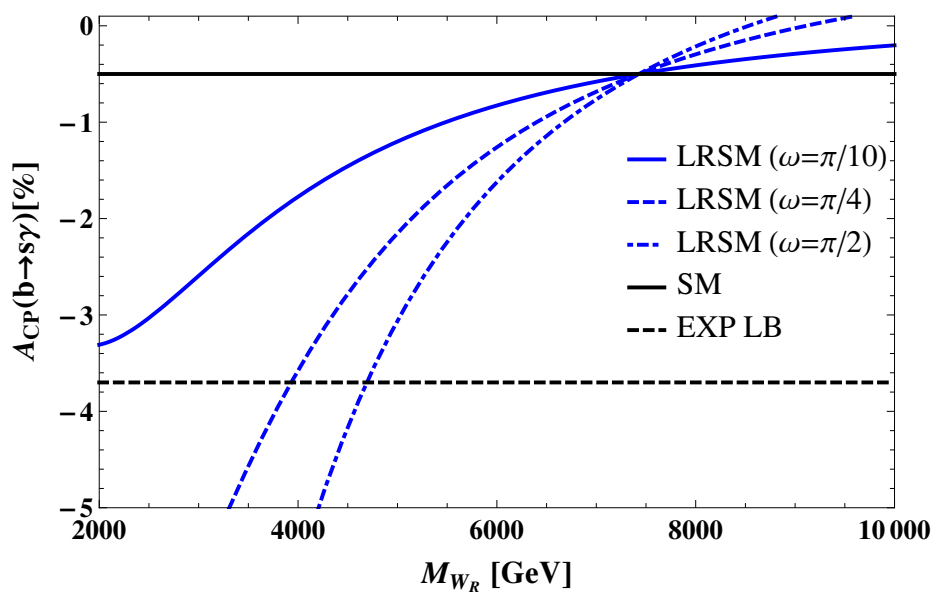

Figure 6. The direct CP asymmetry in $b \rightarrow s \gamma$ in the SM and LRSM which are depicted by the black and blue solid curves. The black dashed line is same as in figure 5 . We also show $\omega=\pi / 4$ and $\pi / 2$ cases by the blue dashed and dashed-dotted curves for comparison, respectively.

the future determination will check NP between $450-750 \mathrm{GeV}$ in this typical SUSY SU(5) with $N_{R}$ case.

\subsubsection{LRSM case}

For the LRSM, $C_{8}^{\mathrm{NP}}$ and $C_{8}^{\prime \mathrm{NP}}$ are

$$
\begin{aligned}
& C_{8}=\rho_{8} \Delta^{L R} C_{8}+\rho_{\mathrm{LR}} \frac{m_{c}}{m_{b}} \sin \zeta \cos \zeta e^{i \alpha} \frac{V_{c b}^{R}}{V_{c b}^{L}}, \\
& C_{8}^{\prime}=\rho_{8} \Delta^{L R} C_{8}^{\prime}+\rho_{\mathrm{LR}} \frac{m_{c}}{m_{b}} \sin \zeta \cos \zeta e^{-i \alpha} \frac{V_{c b}^{R *}}{V_{c b}^{L *}},
\end{aligned}
$$

with

$$
\begin{aligned}
\Delta^{L R} C_{8} & =\frac{m_{t}}{m_{b}} \sin \zeta \cos \zeta e^{i \alpha} \frac{V_{t b}^{R}}{V_{t b}^{L}} f_{\mathrm{LR}}\left(\tilde{x}_{t}\right), \\
\Delta^{L R} C_{8}^{\prime} & =\frac{m_{t}}{m_{b}} \sin \zeta \cos \zeta e^{-i \alpha} \frac{V_{t s}^{R *}}{V_{t s}^{L *}} f_{\mathrm{LR}}\left(\tilde{x}_{t}\right),
\end{aligned}
$$

where $\rho_{8}$ and $\rho_{\mathrm{LR}}$ are the so-called magic number which are given in the ref. [27]. $f_{\mathrm{LR}}(x)$ is also a loop function for the left-right symmetric model given in appendix A.

The asymmetry in the LRSM is shown by the blue curve in figure 6 . We also show $\omega=\pi / 4$ and $\pi / 2$ cases by the blue dashed and dashed-dotted curves for comparison, respectively. One can see from figure 6 that the magnitude of the direct $\mathrm{CP}$ asymmetry in the LRSM is allowed for $M_{\mathrm{NP}} \geq 2 \mathrm{TeV}$ for $\pi / 10$. Hence, the $A_{\mathrm{CP}}$ measurement does not give constraint on the existence of NP in this case at the moment. Furthermore, the future Belle II with $50 \mathrm{ab}^{-1}$ will check the LRSM model up to $3.5 \mathrm{TeV}, 5 \mathrm{TeV}$, and $5.5 \mathrm{TeV}$ for $\omega=\pi / 10, \omega=\pi / 4$, and $\omega=\pi / 2$, respectively. The result is really sensitive to the phase $\omega$ as with SUSY SU(5) with $N_{R}$ case. 


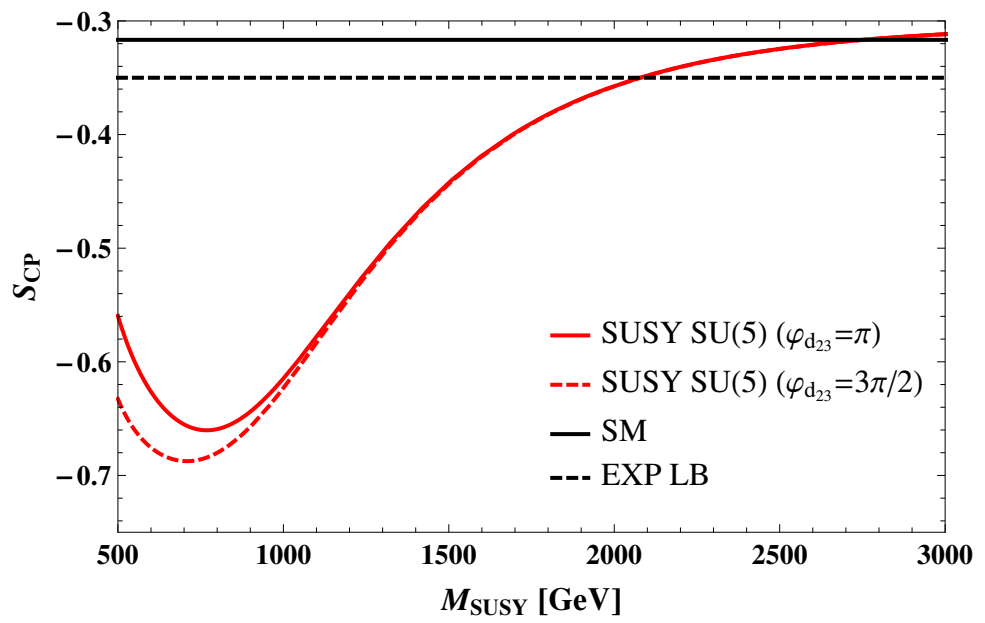

Figure 7. The time-dependent CP asymmetry in $B \rightarrow K_{s} \pi^{0} \gamma$ in the SM and SUSY SU(5) GUT with $N_{R}$, which are depicted by the black and blue solid curves. The black dashed line is the current experimental lower bound [33]. We also show $\varphi_{d_{23}}=3 \pi / 2$ case by the red dashed curve for comparison.

\subsection{Time-dependent CP asymmetry}

We also evaluate the time-dependent $\mathrm{CP}$ asymmetry in the $B \rightarrow K_{s} \pi^{0} \gamma$ decay denoted as $S_{C P}$. The definition of $S_{C P}$ is same in the both model:

$$
S_{\mathrm{CP}}=2 \frac{\operatorname{Im}\left[e^{-2 i \beta_{C K M}} C_{7} C_{7}^{\prime}\right]}{\left|C_{7}\right|^{2}+\left|C_{7}^{\prime}\right|^{2}},
$$

where $2 \beta_{C K M} \approx 43^{\circ}$ is a $\mathrm{CP}$ phase in $B \rightarrow K_{s} \pi^{0} \gamma$ decay.

\subsubsection{SUSY SU(5) with right-handed neutrino case}

We show eq. (3.6) in the SUSY SU(5) with $N_{R}$ and SM cases by red and black solid curves in figure 7 , respectively. The current experimental lower bound as -0.35 is also shown by the black dashed line. We also show $\varphi_{d_{23}}=3 \pi / 2$ case by the red dashed curve for comparison. The current experimental bound seems to exclude up to $2 \mathrm{TeV}$ of the $M_{\mathrm{SUSY}}$ in figure 7 in this naive set up. In other words, the time-dependent CP asymmetry gives the strongest constraint within $\lambda_{\gamma}, A_{\mathrm{CP}}$, and $S_{\mathrm{CP}}$.

\subsubsection{LRSM case}

We show eq. (3.6) in the LRSM and SM cases by blue and black solid curves in figure 8, respectively. The current experimental lower bound constrains the mass of $W_{R}$ up to $7 \mathrm{TeV}$ in this simple set up. The figure shows that the time-dependent CP asymmetry is the strongest constraint even in the LRSM. 


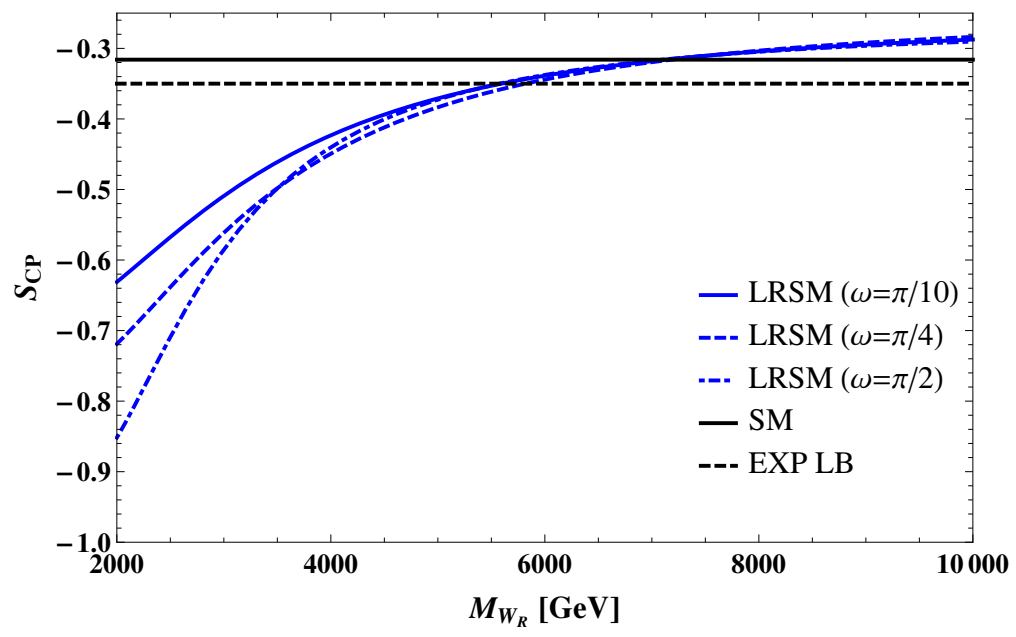

Figure 8. The time-dependent CP asymmetry in $B \rightarrow K_{s} \pi^{0} \gamma$ in the SM and LRSM which are depicted by the black and blue solid curves. The black dashed line is same as in figure 7 . We also show $\omega=\pi / 4$ and $\pi / 2$ cases by the blue dashed and dashed-dotted curves for comparison, respectively.

\section{Comparison between Photon polarization and Measurements of CP asymmetry}

As we mentioned in the Introduction, the photon polarization might become a useful way to determine NP. Actually, the ways to determine the photon chirality by measurement of angular distribution of the final state particles have been discussed in several papers [9-11] (See also $[34,35]$ ). In this paper, we have evaluated three observables: photon polarization, direct CP asymmetry, and time-dependent CP asymmetry. We give some comments on the comparison among the results of those:

- For the determination of photon polarization in the SUSY SU(5) with $N_{R}$ model, the future LHCb experiment with $2 \mathrm{fb}^{-1}$ will be able to check the NP scale up to about $1700 \mathrm{GeV}$, which corresponds to $M_{2} \simeq 1400 \mathrm{GeV}$ and $M_{\tilde{g}} \simeq 4900 \mathrm{GeV}$, at the typical point of this model.

- For the measurement of $A_{\mathrm{CP}}$ in the SUSY SU(5) with $N_{R}$ model, the experiment does not currently constrain on the NP scale even if one takes the maximally allowed CP phase as $\varphi_{d_{23}}=3 \pi / 2$. The future Belle II with $50 \mathrm{ab}^{-1}$ will check NP between $450-750 \mathrm{GeV}$.

- For the determination of photon polarization in the LRSM, the future LHCb experiment will check the NP scale up to $3.7 \mathrm{TeV}$ in this case.

- For the measurement of $A_{\mathrm{CP}}$ in the LRSM, the experiment constrain up to $4.7 \mathrm{TeV}$ depending on the phase $\omega$. Furthermore, the future Belle II with $50 \mathrm{ab}^{-1}$ will check up to $5.5 \mathrm{TeV}$ when $\omega$ maximize the direct $\mathrm{CP}$ asymmetry. On the other hand, the future experiment reach $3.5 \mathrm{TeV}$ at most when $\omega$ minimize the direct $\mathrm{CP}$ asymmetry. 
- The time-dependent CP asymmetry is the most stringent constraint in both models and this CP asymmetry does not depend on CP asymmetry parameters so much.

- Thus, we mention that there is a region where the determination of photon polarization is more ascendant for the NP search than that of direct CP asymmetry in both models. However, time-dependent $\mathrm{CP}$ asymmetry always gives us more stringent constraint than other observables.

\section{Summary}

One might be able to obtain the existence of NP and discriminate among the SM and NP if one can precisely determine the chirality of the $s$ quark in the $b \rightarrow s \gamma$ process. The chirality of $s$ quark can be determined by measuring the polarization of photon in the process. And, there are several ways to measure the photon polarization. In addition to the determination of photon polarization, the observation of $\mathrm{CP}$ asymmetry in the process is still sensitive to the existence of NP. Thus, simultaneous studies of photon polarization and CP asymmetry in the $b \rightarrow s \gamma$ process will be intriguing for the experimental search of NP.

We have investigated the $b \rightarrow s \gamma$ process in the SUSY SU(5) GUT with $N_{R}$ model and the LRSM. The ratio $\left|C_{7}^{\prime} / C_{7}\right|$, the polarization parameter of photon, and the direct $\mathrm{CP}$ asymmetry in the process have been evaluated in both models. The time-dependent CP asymmetry seems to be the best way to find the NP effect. However, the combination of $\mathrm{CP}$ violation and photon polarization can discriminate NP beyond the SM at the end. Furthermore, there might be a region where the determination of photon polarization is more sensitive for the new physics search than that of CP asymmetry.

\section{Acknowledgments}

We would like to thank to Y. Okada for fruitful discussion. This work is partially supported by Scientific Grant by Ministry of Education and Science, No. 24540272 and Grant-in-Aid for Scientific Research on Innovative Areas titled "Unification and Development of the Neutrino Science Frontier", No. 25105001. The work of R.T. are supported by Research Fellowships of the Japan Society for the Promotion of Science for Young Scientists.

\section{Loop functions}

We give loop functions [18] which are utilized in our analyses:

$$
\begin{aligned}
h_{7}(x) & =-\frac{5 x^{2}-3 x}{12(1-x)^{2}}-\frac{3 x^{2}-2 x}{6(1-x)^{3}} \log x, \\
h_{8}(x) & =-\frac{x^{2}-3 x}{4(1-x)^{2}}+\frac{x}{2(1-x)^{3}} \log x, \\
f_{7,8}^{(1)}(x, y) & =\frac{2}{x-y}\left(f_{7,8}^{(2)}(x)-f_{7,8}^{(2)}(y)\right), \\
f_{7}^{(2)}(x) & =-\frac{13-7 x}{24(1-x)^{3}}-\frac{3+2 x-2 x^{2}}{12(1-x)^{4}} \log x,
\end{aligned}
$$




$$
\begin{aligned}
f_{8}^{(2)}(x) & =\frac{1+5 x}{8(1-x)^{3}}+\frac{x(2+x)}{4(1-x)^{4}} \log x, \\
f_{\mathrm{LR}}(x) & =-\frac{x^{2}+x+4}{4(1-x)^{2}}-\frac{3 x}{2(1-x)^{2}} \log x, \\
g_{7}^{(1)}(x) & =-\frac{2(1+5 x)}{9(1-x)^{3}}-\frac{4 x(1+x)}{3(1-x)^{5}} \log x, \\
g_{8}^{(1)}(x) & =\frac{11+x}{3(1-x)^{3}}+\frac{9+16 x-x^{2}}{6(1-x)^{4}} \log x, \\
g_{7}^{(2)}(x) & =-\frac{2\left(1+10 x+x^{2}\right)}{9(1-x)^{4}}-\frac{4 x(1+x)}{3(1-x)^{5}} \log x, \\
g_{8}^{(2)}(x) & =\frac{53+44 x-x^{2}}{12(1-x)^{4}}+\frac{3+11 x+2 x^{2}}{2(1-x)^{5}} \log x, \\
D_{0}^{\prime}(x) & =\frac{-8 x^{3}-5 x^{2}+7 x}{24(x-1)^{3}}+\frac{3 x^{3}-2 x^{2}}{4(x-1)^{4}} \log x, \\
A_{\mathrm{LR}}(x) & =\frac{-5 x^{2}+31 x-20}{6(x-1)^{2}}-\frac{3 x^{2}-2 x}{(x-1)^{3}} \log x, \\
A_{H}^{2}(x) & =\frac{22 x^{3}-53 x^{2}+25 x}{72(x-1)^{3}}-\frac{3 x^{3}-8 x^{2}+4 x}{12(x-1)} \log x .
\end{aligned}
$$

\section{$7 \quad$ Mass insertion parameters}

The mass insertion parameters are defined as (e.g., see [36])

$$
\begin{aligned}
\left(\delta_{q}^{X X}\right)_{i j} & \equiv \frac{\left(m_{\tilde{q}_{X}}^{2}\right)_{i j}}{\tilde{m}_{\tilde{f}}^{2}} \\
\left(\delta_{d}^{X Y}\right)_{i j} & \equiv \frac{v_{d}\left(A_{d}-\mu t_{\beta}\right)_{i j}}{\tilde{m}_{\tilde{f}}^{2}}, \\
\left(\delta_{u}^{X Y}\right)_{i j} & \equiv \frac{v_{u}\left(A_{u}-\mu \cot \right)_{i j}}{\tilde{m}_{\tilde{f}}^{2}},
\end{aligned}
$$

with $X \neq Y$ where $\tilde{m}_{\tilde{f}}^{2}(\tilde{f}=\tilde{u}, \tilde{d})$ denotes up- and down-type averaged squark mass, and the numerator of eq. (7.1) can be written as

$$
\begin{aligned}
& \left(m_{\tilde{u}_{L}}^{2}\right)_{i j} \simeq-V_{3 i} V_{3 j}^{*} \frac{f_{t}^{2}}{(4 \pi)^{2}}\left(3 m_{0}^{2}+A_{0}^{2}\right)\left(2 \log \frac{M_{\mathrm{pl}}^{2}}{M_{H_{c}}^{2}}+\log \frac{M_{H_{c}}^{2}}{M_{\mathrm{SUSY}}^{2}}\right), \\
& \left(m_{\tilde{u}_{R}}^{2}\right)_{i j} \simeq-e^{-i \varphi_{u_{i j}}} V_{3 i}^{*} V_{3 j} \frac{2 f_{b}^{2}}{(4 \pi)^{2}}\left(3 m_{0}^{2}+A_{0}^{2}\right) \log \frac{M_{\mathrm{pl}}^{2}}{M_{H_{c}}^{2}}, \\
& \left(m_{\tilde{d}_{L}}^{2}\right)_{i j} \simeq-V_{i 3}^{*} V_{j 3} \frac{2 f_{t}^{2}}{(4 \pi)^{2}}\left(3 m_{0}^{2}+A_{0}^{2}\right)\left(3 \log \frac{M_{\mathrm{pl}}^{2}}{M_{H_{c}}^{2}}+\log \frac{M_{H_{c}}^{2}}{M_{\mathrm{SUSY}}^{2}}\right), \\
& \left(m_{\tilde{d}_{R}}^{2}\right)_{i j} \simeq-e^{-i \varphi_{d_{i j}}} U_{k i}^{*} V_{k j} \frac{f_{\nu_{k}}^{2}}{(4 \pi)^{2}}\left(3 m_{0}^{2}+A_{0}^{2}\right) \log \frac{M_{\mathrm{pl}}^{2}}{M_{H_{c}}^{2}} .
\end{aligned}
$$

Here, $m_{0}$ and $A_{0}$ are the universal scalar mass and trilinear coupling, respectively. 
Open Access. This article is distributed under the terms of the Creative Commons Attribution License (CC-BY 4.0), which permits any use, distribution and reproduction in any medium, provided the original author(s) and source are credited.

\section{References}

[1] R. Barbieri and L.J. Hall, Signals for supersymmetric unification, Phys. Lett. B 338 (1994) 212 [hep-ph/9408406] [INSPIRE].

[2] R. Barbieri, L.J. Hall and A. Strumia, Violations of lepton flavor and CP in supersymmetric unified theories, Nucl. Phys. B 445 (1995) 219 [hep-ph/9501334] [INSPIRE].

[3] Y. Kuno and Y. Okada, Proposed $\mu \rightarrow$ ey search with polarized muons, Phys. Rev. Lett. 77 (1996) 434 [hep-ph/9604296] [INSPIRE].

[4] M. Gronau, Y. Grossman, D. Pirjol and A. Ryd, Measuring the photon polarization in $B \rightarrow K \pi \pi \gamma$, Phys. Rev. Lett. 88 (2002) 051802 [hep-ph/0107254] [InSPIRE].

[5] M. Gronau and D. Pirjol, Photon polarization in radiative $B$ decays, Phys. Rev. D 66 (2002) 054008 [hep-ph/0205065] [INSPIRE].

[6] B. Grinstein, Y. Grossman, Z. Ligeti and D. Pirjol, The photon polarization in $B \rightarrow X \gamma$ in the standard model, Phys. Rev. D 71 (2005) 011504 [hep-ph/0412019] [INSPIRE].

[7] Y.Y. Keum, M. Matsumori and A.I. Sanda, CP asymmetry, branching ratios and isospin breaking effects of $B \rightarrow K^{*} \gamma$ with perturbative $Q C D$ approach, Phys. Rev. D 72 (2005) 014013 [hep-ph/0406055] [INSPIRE].

[8] LHCb collaboration, Observation of photon polarization in the $b \rightarrow s \gamma$ transition, Phys. Rev. Lett. 112 (2014) 161801 [arXiv:1402.6852] [INSPIRE].

[9] D. Melikhov, N. Nikitin and S. Simula, Probing right-handed currents in $B \rightarrow K^{*} \ell^{+} \ell^{-}$ transitions, Phys. Lett. B 442 (1998) 381 [hep-ph/9807464] [INSPIRE].

[10] C.S. Kim, Y.G. Kim, C.-D. Lu and T. Morozumi, Azimuthal angle distribution in $B K^{*}(\rightarrow K p i) \ell^{+} \ell^{-}$at low invariant $m_{\ell^{+} \ell^{-}}$region, Phys. Rev. D 62 (2000) 034013 [hep-ph/0001151] [INSPIRE].

[11] D. Becirevic, E. Kou, A. Le Yaouanc and A. Tayduganov, Future prospects for the determination of the Wilson coefficient $C_{7 \gamma}^{\prime}$, JHEP 08 (2012) 090 [arXiv:1206.1502] [INSPIRE].

[12] J.M. Soares, CP violation in radiative b decays, Nucl. Phys. B 367 (1991) 575 [InSPIRE].

[13] M. Endo and N. Yokozaki, Large CP-violation in $B_{s}$ Meson Mixing with EDM constraint in Supersymmetry, JHEP 03 (2011) 130 [arXiv: 1012.5501] [INSPIRE].

[14] A.J. Buras, Weak Hamiltonian, CP-violation and rare decays, hep-ph/9806471 [INSPIRE].

[15] A.L. Kagan and M. Neubert, QCD anatomy of $B \rightarrow X_{s \gamma}$ decays, Eur. Phys. J. C 7 (1999) 5 [hep-ph/9805303] [INSPIRE].

[16] M. Ciuchini, E. Franco, G. Martinelli, L. Reina and L. Silvestrini, Scheme independence of the effective Hamiltonian for $b \rightarrow s \gamma$ and $b \rightarrow s g$ decays, Phys. Lett. B 316 (1993) 127 [hep-ph/9307364] [INSPIRE].

[17] A.J. Buras, M. Misiak, M. Münz and S. Pokorski, Theoretical uncertainties and phenomenological aspects of $B \rightarrow X_{s \gamma}$ decay, Nucl. Phys. B 424 (1994) 374 [hep-ph/9311345] [INSPIRE]. 
[18] W. Altmannshofer, A.J. Buras, S. Gori, P. Paradisi and D.M. Straub, Anatomy and Phenomenology of FCNC and CPV Effects in SUSY Theories, Nucl. Phys. B 830 (2010) 17 [arXiv: 0909.1333] [INSPIRE].

[19] Particle Data Group collaboration, K.A. Olive et al., Review of particle physics, Chin. Phys. C 38 (2014) 090001.

[20] M.C. Gonzalez-Garcia, M. Maltoni and T. Schwetz, Updated fit to three neutrino mixing: status of leptonic CP-violation, JHEP 11 (2014) 052 [arXiv:1409.5439] [INSPIRE].

$[21]$ E. Kou, A. Le Yaouanc and A. Tayduganov, Determining the photon polarization of the $b \rightarrow s \gamma$ using the $B \rightarrow K_{1}(1270) \gamma \rightarrow(K \pi \pi) \gamma$ decay, Phys. Rev. D 83 (2011) 094007 [arXiv: 1011.6593] [INSPIRE].

[22] J.C. Pati and A. Salam, Lepton Number as the Fourth Color, Phys. Rev. D 10 (1974) 275 [Erratum ibid. D 11 (1975) 703-703] [INSPIRE].

[23] R.N. Mohapatra and J.C. Pati, Left-Right Gauge Symmetry and an Isoconjugate Model of CP-violation, Phys. Rev. D 11 (1975) 566 [INSPIRE].

[24] R.N. Mohapatra and J.C. Pati, A Natural Left-Right Symmetry, Phys. Rev. D 11 (1975) 2558 [inSPIRE].

[25] G. Senjanović and R.N. Mohapatra, Exact Left-Right Symmetry and Spontaneous Violation of Parity, Phys. Rev. D 12 (1975) 1502 [inSPIRE].

[26] U. Amaldi et al., A Comprehensive Analysis of Data Pertaining to the Weak Neutral Current and the Intermediate Vector Boson Masses, Phys. Rev. D 36 (1987) 1385 [InSPIRE].

[27] M. Blanke, A.J. Buras, K. Gemmler and T. Heidsieck, $\Delta F=2$ observables and $B \rightarrow X_{q} \gamma$ decays in the left-right model: Higgs particles striking back, JHEP 03 (2012) 024 [arXiv:1111.5014] [INSPIRE].

[28] E. Kou, C.-D. Lü and F.-S. Yu, Photon Polarization in the $b \rightarrow s \gamma$ processes in the Left-Right Symmetric Model, JHEP 12 (2013) 102 [arXiv:1305.3173] [INSPIRE].

[29] Y. Zhang, H. An, X. Ji and R.N. Mohapatra, General CP-violation in Minimal Left-Right Symmetric Model and Constraints on the Right-Handed Scale, Nucl. Phys. B 802 (2008) 247 [arXiv: 0712.4218] [INSPIRE].

[30] G. Senjanović, Spontaneous Breakdown of Parity in a Class of Gauge Theories, Nucl. Phys. B 153 (1979) 334 [InSPIRE].

[31] M.A.B. Beg, R.V. Budny, R.N. Mohapatra and A. Sirlin, Manifest Left-Right Symmetry and Its Experimental Consequences, Phys. Rev. Lett. 38 (1977) 1252 [Erratum ibid. 39 (1977) 54] [INSPIRE].

[32] T. Hurth, E. Lunghi and W. Porod, Untagged $\bar{B} \rightarrow X_{s+d} \gamma$ CP asymmetry as a probe for new physics, Nucl. Phys. B 704 (2005) 56 [hep-ph/0312260] [INSPIRE].

[33] Quark Flavor Physics Working Group collaboration, J.N. Butler et al., Working Group Report: Quark Flavor Physics, arXiv:1311.1076 [INSPIRE].

[34] R.-H. Li, C.-D. Lu and W. Wang, Branching ratios, forward-backward asymmetries and angular distributions of $B \rightarrow K_{2}^{*} l^{+} l^{-}$in the standard model and new physics scenarios, Phys. Rev. D 83 (2011) 034034 [arXiv: 1012.2129] [INSPIRE].

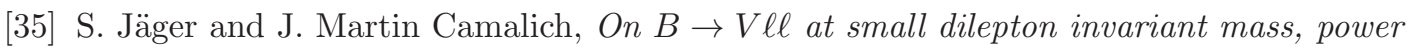
corrections and new physics, JHEP 05 (2013) 043 [arXiv: 1212.2263] [INSPIRE].

[36] J. Hisano, M. Kakizaki, M. Nagai and Y. Shimizu, Hadronic EDMs in SUSY SU(5) GUTs with right-handed neutrinos, Phys. Lett. B 604 (2004) 216 [hep-ph/0407169] [INSPIRE]. 